\title{
Comparative trial of Co-trimoxazole and Chloramphenicol in Typhoid Fever
}

\author{
H. V. SARDESAI, R. S. KARANDIKAR, R. G. HARSHE
}

British Medical fournal, 1973, 1, 82-83

\section{Summary}

A comparative trial of co-trimoxazole and chloramphenicol was conducted in two groups of 50 patients each to try to resolve conflicting opinions on the relative merits of the two drugs in the treatment of typhoid fever. We conclude that in our part of India co-trimoxazole is superior to chloramphenicol and that differences in our findings to those of others may perhaps be accounted for by differences in strains of Salmonella typhi, ethnic differences, and possibly differences in herd immunity to typhoid.

\section{Introduction}

Comparative clinical studies of co-trimoxazole (trimethoprimsulphamethoxazole) and chloramphenicol in enteric fever in different parts of the world have given conflicting results, some indicating that co-trimoxazole is superior to chloramphenicol (Kamat, 1970) and others the opposite (Scragg and Rubidge, 1971). To try to resolve the issue we undertook a comparative clinical trial of the two drugs.

\section{Patients and Methods}

The trial was conducted in the Sassoon Hospitals, Poona, and was restricted to inpatients over 12 years of age for better control and standardization of dosage and follow-up.

The following investigations were carried out in patients suspected to be suffering from enteric fever: routine urine and blood examinations, blood-clot culture for salmonella, and Widal reaction. No specific therapy was given for 96 hours, by which time the result of the blood-clot culture was available. Only patients whose culture was positive for Salmonella typhi were admitted to the trial. They were allotted to one of two treatment groups on a randomized basis, the control group receiving chloramphenicol and the trial group receiving cotrimoxazole. Antipyretics were not given since they might have affected fever, one of the criteria for assessment. Intravenous fluids were given when indicated. The patients were examined daily.

The response to specific therapy was assessed on the following criteria: (1) the duration of fever; (2) the duration of toxic symptoms such as severe headache, mental confusion, disorientation, delirium, and involuntary movements; (3) exacerbation of toxaemia after starting specific therapy (toxic crises); (4) relapse of fever within three weeks of defervescence; and (5) occurrence of complications of typhoid fever.

Patients in the trial group were given four tablets of cotrimoxazole (each containing trimethoprim $80 \mathrm{mg}$ and sulphamethoxazole $400 \mathrm{mg}$ ) twice daily until defervescence, and then two tablets twice daily for seven days. Patients in the control group were given chloramphenicol two capsules six-hourly until defervescence and then one capsule six-hourly for 14 days, each capsule containing $250 \mathrm{mg}$.

Sassoon Hospitals, Poona, India

H. V. SARDESAI, M.D., M.R.C.P., Honorary Physician

R. S. KARANDIKAR, M.D., Registrar in Medicine

R. G. HARSHE, M.D., House Physician
There were 50 patients in each group, the trial group consisting of $\mathbf{4 0}$ males and 10 females and the control group of 37 males and 13 females. The mean age of patients in the trial group was 23.44 years (range 13-45) and in the control group 24.6 years (range 11-75). Their distribution among age groups is shown in table $I$.

TABLE I-Age-group Distribution of Patients

\begin{tabular}{c|c|c}
\hline Age & No. in Trial Group & No. in Control Group \\
\hline $12-20$ & 20 & 21 \\
$21-30$ & 21 & 19 \\
$31-40$ & 6 & 7 \\
$41-50$ & 3 & 1 \\
Over 50 & - & 2 \\
\hline
\end{tabular}

Most patients had an axillary temperature on admission of from $100^{\circ}$ to $102^{\circ} \mathrm{F}\left(37.8^{\circ}-38.9^{\circ} \mathrm{C}\right)$ but in 11 in the trial group and in 13 in the control group it exceeded $102^{\circ} \mathrm{F}$. The duration of fever before admission is shown in table II. The mean

TABLE II-Duration of Fever before Admission

\begin{tabular}{c|c|c}
\hline \multirow{2}{*}{$\begin{array}{c}\text { Duration } \\
\text { (Days) }\end{array}$} & Trial Group & No. of Patients \\
\hline $1-3$ & 4 & Control Group \\
$4-6$ & 16 & 7 \\
$7-9$ & 16 & 18 \\
Over 9 & 14 & 11 \\
\hline
\end{tabular}

duration of fever was 6.98 days in the control and 7.53 days in the trial group. Thirty-two of the patients in the trial group and 36 of those in the control group were toxaemic at the time of starting specific therapy.

\section{Results}

All patients in both groups responded to treatment. There was no significant difference in the response of fever to treatment in either group. The mean period of fever before defervescence was 4.55 days in the control group and 4.98 days in the trial group. Toxaemia responded to treatment earlier in those treated with co-trimoxazole (trial group) than in those treated with chloramphenicol (control group) (table III).

TABLE III-Response of Toxaemia to Treatment

\begin{tabular}{c|c|c}
\hline \multirow{2}{*}{$\begin{array}{c}\text { Duration of Treatment } \\
\text { before Response } \\
\text { (Days) }\end{array}$} & \multicolumn{2}{|c}{ No. of Patients } \\
\cline { 2 - 3 } & Trial Group $(\mathrm{n}=32)$ & Control Group $(\mathrm{n}=36)$ \\
\hline $1-3$ & 25 & 18 \\
$4-6$ & 6 & 12 \\
Over 6 & 1 & 6 \\
\hline
\end{tabular}

A toxic crisis-drug-induced exacerbation of toxaemia-was seen in two patients in the control group but in none in the trial group. Two patients in the control group relapsed. One developed fever with a positive blood culture 17 days after completing a course of chloramphenicol treatment. He responded to co-trimoxazole in four days and had no further relapse. The other patient who relapsed developed fever and a positive 
blood culture 16 days after completing a course of chloramphenicol. He also responded to co-trimoxazole in four days and had no further relapse. Both these patients may have been reinfected, but far more probably they were genuine cases of relapse.

There were no serious complications such as haemorrhage, perforation, or circulatory collapse in any patient in either group. Nor were there any severe side effects. Minor side effects were equally common in both groups, the only notable difference being a significantly greater incidence of weakness in the control group (19 patients) compared with the trial group (5 patients).

\section{Discussion}

All patients in both groups in our series responded to specific therapy, thus confirming the results of Akinkugbe et al. (1968), Farid et al. (1970), and Kamat (1970). In contrast, two out of 23 patients in Geddes et al. (1971) series and 8 out of 103 in Scragg and Rubidge's (1971) series treated with co-trimoxazole failed to respond. Farid's (1971) observations on the dosage in Scragg and Rubidge's series that underweight and undernourished children who were given co-trimoxazole on a bodyweight basis might have received less than a therapeutic dose is very pertinent and may explain Scragg and Rubidge's poor results with the drug. This also confirms our earlier experience (Sardesai, 1971) when we did not encounter a single failure in 40 patients treated with co-trimoxazole.

In this trial the response of toxaemia to treatment occurred significantly earlier in the trial (co-trimoxazole) group compared with the control (chloramphenicol) group. Before the end of the third day $78.1 \%$ of toxaemic patients in the trial group became non-toxaemic compared with only $50 \%$ in the control group. At the end of six days only $3.1 \%$ of patients in the trial group remained toxaemic as compared to $16.6 \%$ in the control group. These results are similar to almost all previous workers (Kamat, 1969; Kamat, 1970; Farid et al., 1970; Geddes et al., 1971; Scragg and Rubidge, 1971).
Two patients in our control group relapsed but none in the trial group did so. Kamat (1970), and Farid et al. (1970) did not encounter any relapse in patients treated with cotrimoxazole. In Geddes et al. (1971) series there were two relapses out of 23 patients and in Scragg and Rubidge's series a staggering relapse rate of $12.6 \%$ in patients treated with cotrimoxazole.

Our overall results are similar to those in the very large series of Kamat (1970) in that co-trimoxazole seemed superior to chloramphenicol in relieving toxaemia but equal to it when judged by the duration of fever. In our series, however, cotrimoxazole also seemed to be superior to chloramphenicol when judged by the incidence of relapse. The comparatively poorer results with co-trimoxazole in the series of Geddes et al. (1971) and Scragg and Rubidge (1971) are difficult to explain. It seems that various factors such as differences in strains of Salm. typhi, ethnic differences, and possibly differences in herd immunity to typhoid may be responsible for the apparent contradictions. Clearly, however, in our part of India cotrimoxazole is superior to chloramphenicol in the treatment of typhoid fever.

We are grateful to Dr. V. S. Ganla, dean, Sassoon Group of Hospitals and B. J. Medical College, for his kind permission to carry out the study. We are also grateful to Burroughs Wellcome and Co. (India) Pvt. Ltd., for the supply of tablets for the trial.

\section{References}

Akinkugbe, O. O. et al. (1968). British Medical fournal, 3, 721.

Farid, Z. et al. (1970). British Medical fournal, 3, 323.

Farid, Z. (1971). British Medical fournal, 4, 364.

Geddes, A. M. et al. (1971). British Medical fournal, 3, 451.

Kamat, S. A. (1969). Proceedings of the Sixth International Congress on Chemotherapy, Tokyo.

Kamat, S. A. (1970). British Medical fournal, 3, 320.

Sardesai, H. V. (1971). Transactions of the Royal Society of Tropical Medicine and Hygiene, 65, 189.

Scragg, J. N. and Rubidge, C. J. (1971). British Medical fournal, 3, 738.

\title{
Association between Previous Tuberculous Infection and Cerebal Glioma
}

\author{
DONALD W. WARD, M. L. MATTISON, RONALD FINN
}

British Medical fournal, 1973, 1, 83-84

\section{Summary}

An increased incidence of previous infection with tuberculosis has been found in a series of patients with cerebral gliomas, and it is suggested that such an association may be due to defective immunity acting as a common aetiological factor.

\section{Introduction}

When it was noticed that three patients with cerebral gliomas, seen consecutively, had associated disease a survey of further patients with gliomas was conducted (Finn et al., 1972). This showed that about a quarter had a history or significant radio-

Royal Southern Hospital, Liverpool L8 5SH

DONALD W. WARD, M.B., M.R.C.P., Senior Medical Registrar

M. L. MATTISON, M.B., ch.B., House Physician

RONALD FINN, M.D., F.R.C.P., Consultant Physician logical evidence of previous tuberculous infection compared with a tenth of a series of 56 controls. Consequently, the larger study was carried out.

\section{Patients and Methods}

For the purpose of this investigation previous tuberculous infection was defined as either a previous history of clinical tuberculosis or the presence on chest radiography of healed apical fibrotic lesions, with or without extensive calcification, often involving the hilar regions.

Altogether, 100 case records of patients, each with a histologically-proved glioma, were studied. Eight had to be rejected because of inadequate information. The ages of the remaining 92 patients ranged from the mid-20s to over 80 years.

As controls we examined in detail the previous histories and chest radiographs of 100 patients admitted consecutively to two general medical units. In view of the decline in tuberculous infection over recent years we limited selection to patients aged 50 years or over, with the exception of three under the age of 50 who were known to have had tuberculosis in the past. 